\title{
WIRELESS ENERGY SUPPLY TO AIRCRAFT STRUCTURAL HEALTH MONITORING NODES USING ULTRASONIC LAMB WAVES
}

\author{
Aleksander Kural \\ Institute of Aviation, Al. Krakowska 110/114, 02-256 Warsaw, Poland \\ Aleksander.Kural@ge.com
}

\begin{abstract}
This article is based on research done during the author's PhD at Cardiff University, UK. A prototype of a novel wireless energy transmission system aimed at the use with wireless aircraft structural health monitoring (SHM) sensor nodes is described. The system uses ultrasonic guided plate waves (Lamb waves) to transmit energy along an aluminium plate, similar to those used in aircraft structures. Three types of piezoelectric transducers generating and receiving the ultrasonic vibration were compared. The Smart Material MFC M8528-P1 was found to achieve the best performance, allowing the transmission of $17 \mathrm{~mW}$ across a $54 \mathrm{~cm}$ distance, while being driven with a $20 \mathrm{~V}$ signal. Laser vibrometer imaging and LISA software simulation of the Lamb wave propagation in the experimental plate were also performed. Based on these, ideas for a further development of the system were proposed.
\end{abstract}

Keywords: Structural Health Monitoring, wireless ultrasonic energy transmission system, transmission transducers tests

\section{INTRODUCTION}

The experimental energy transmission system described in this article was originally aimed at the use with wireless sensor nodes of an aircraft structural health (SHM) monitoring system. Such systems are at present under investigation, among others, by the Cardiff University School of Engineering [1]. The system is to consist of a number of ultrasonic damage detection sensors distributed over the structure of the aircraft. Due to aircraft weight and assembly constraints, the sensors should be designed to work without wired connections. This requires a wireless data transmission and an autonomous energy supply solution to be developed.

Currently the power requirement of a sensor node is estimated at $170 \mathrm{~mW}$ during operation and $50 \mathrm{~mW}$ in sleep mode, although these values are expected to decrease as the development of the technology progresses. For the purpose of the autonomous energy supply, various types of batteries as well as vibration and thermal energy harvesting devices are considered. Due to regulatory and life time considerations batteries are usually ruled out in aircraft applications. Energy harvesting devices, on the other hand, may not be able to provide sufficient power during all phases of aircraft operation.

In an attempt to overcome the above shortcomings, a wireless energy transmission system was proposed. The proposed system uses ultrasonic guided plate waves (Lamb waves) propagating through the aircraft structure to transmit energy to SHM sensor nodes. A vibration generator ("transmitter") is to be positioned near an exiting power supply line in the aircraft and be supplied with energy from this line. The vibration will then propagate through the structure in the form of plate waves. Upon reaching a sensor node, the incoming vibration will drive a piezoelectric 
transducer ("receiver") and generate electricity which will be used to power the sensor electronics (Figure 1).

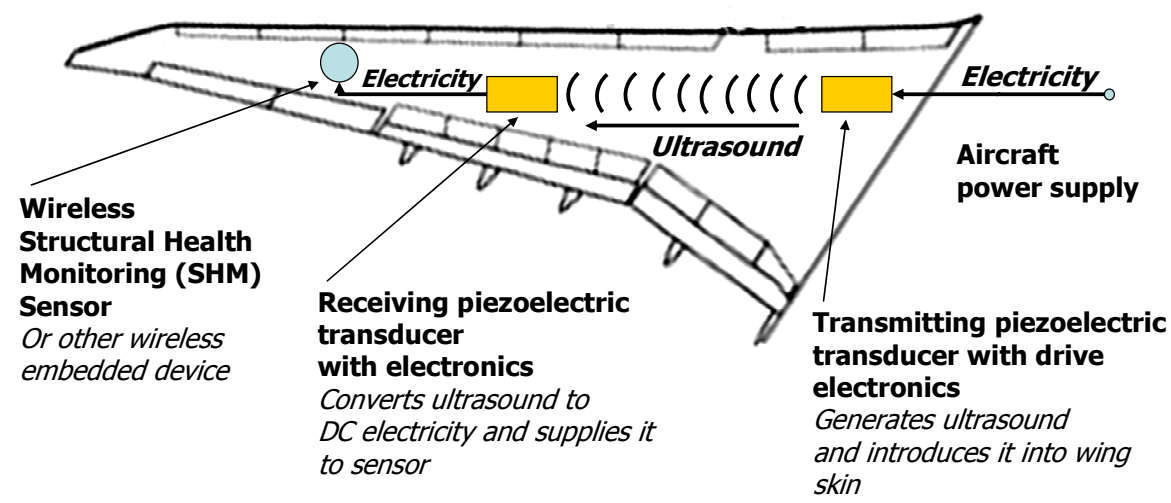

\section{Figure 1. Illustration of the concept of the ultrasonic energy transmission system for aircraft SHM sensors}

\section{REVIEW OF PREVIOUS ULTRASONIC ENERGY TRANSMISSION DEVELOPMENTS}

Energy transmission using ultrasound has been researched previously. Published work concentrated on the transmission through the thickness of a solid material with the use of bulk acoustic waves. Setups aimed at applications in mechanical engineering were reported by several research groups [2] - [6]. Typically the application was the power supply to sensors located inside pressure vessels without the need to breach the vessel wall. Applications in the medical engineering field, aimed at supplying power through tissue to implants were also reported [7], [8]. The power throughputs ranged from $30 \mathrm{~mW}$ to $1 \mathrm{~kW}$ in mechanical engineering applications and from 0.002 to $0.2 \mathrm{~mW}$ in medical engineering applications. Transmission distances in all applications were not higher than $150 \mathrm{~mm}$. More recently a start-up company began working on a wireless charging solution for mobile consumer devices [9]. The proposed system is to use focused beams of ultrasound propagating through air at distances of several metres.

\section{LAMB WAVE ENERGY TRANSMISSION SYSTEM CONCEPT}

The SHM power supply application requires energy transmission with throughputs on the order of $100 \mathrm{~mW}$ across distances on the order of one to several metres. Because of the required distance, the previously developed bulk acoustic wave-based systems cannot be directly applied. The energy transmission system described in this article takes advantage of the fact that aircraft are built of plate structures. Acoustic vibration propagates in plates in the form of a particular type of a guided wave, known as the guided plate wave (or Lamb wave, after the researcher who first described it) [10]. This type of wave, as shown in this article, is characterised by a low attenuation over distance. This makes it particularly suitable as a medium for energy transmission.

\section{EXPERIMENTAL LAMB WAVE ENERGY TRANSMISSION SETUP}

The aim of the research described in this article was to build a proof-of-concept Lamb wave energy transmission system, understand factors influencing its performance and identify the best performing types of piezoelectric transducers to be used as transmitters and receivers of guided plate waves.

The experimental system was built on a $1000 \times 820 \mathrm{~mm}, 1.5 \mathrm{~mm}$ thick aluminium plate. The distance between the transmitting and the receiving transducers was $535 \mathrm{~mm}$ (Figure 2). 


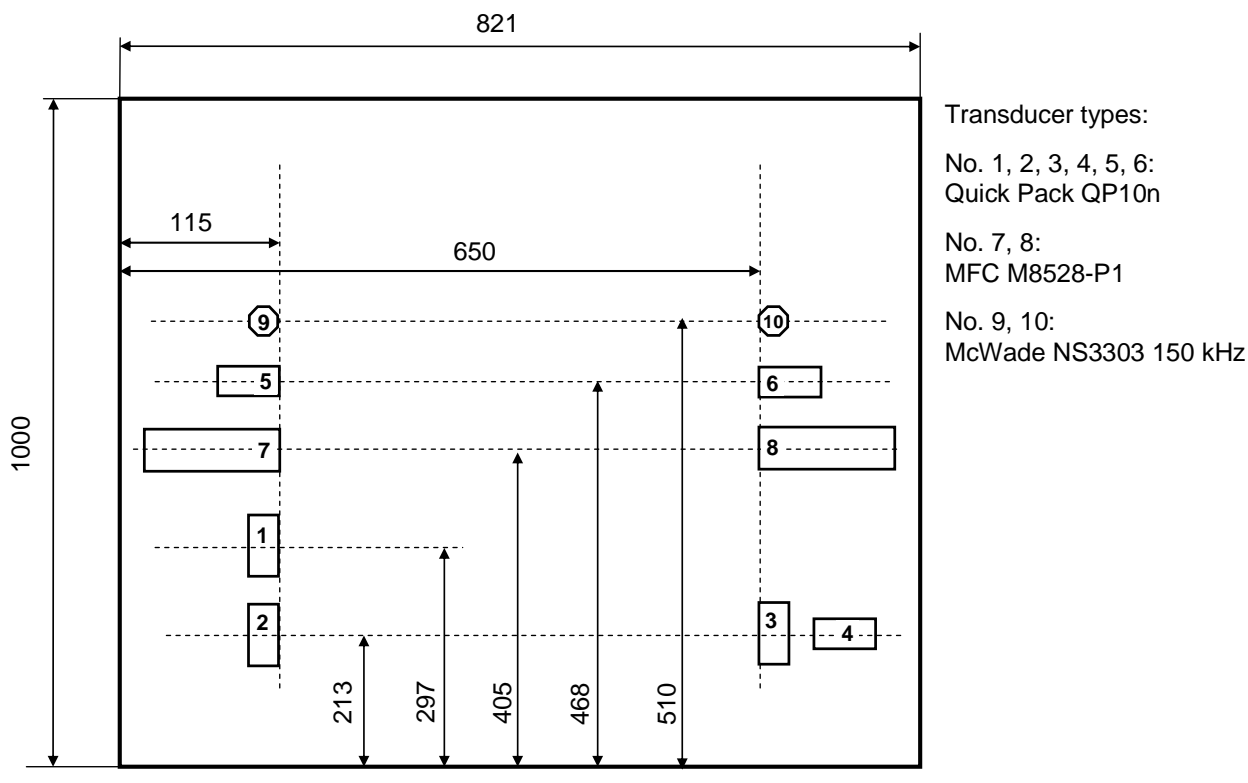

Figure 2. Dimensions of the experimental aluminium plate and locations of transducers

Three transducer types were investigated. The McWade $150 \mathrm{kHz}$ transducers had originally been developed as aviation acoustic emission SHM sensors [11]. It is made of a $\sim 18 \mathrm{~mm}$ diameter $x \sim 10 \mathrm{~mm}$ tall lead zirconate titanate (PZT) crystal enclosed in a brass case. The size of the crystal results in well-defined resonance frequencies at 117 and $150 \mathrm{kHz}$, and a narrow band (of several $\mathrm{kHz}$ ) around the $117 \mathrm{kHz}$ resonance within which the transducer operates efficiently.

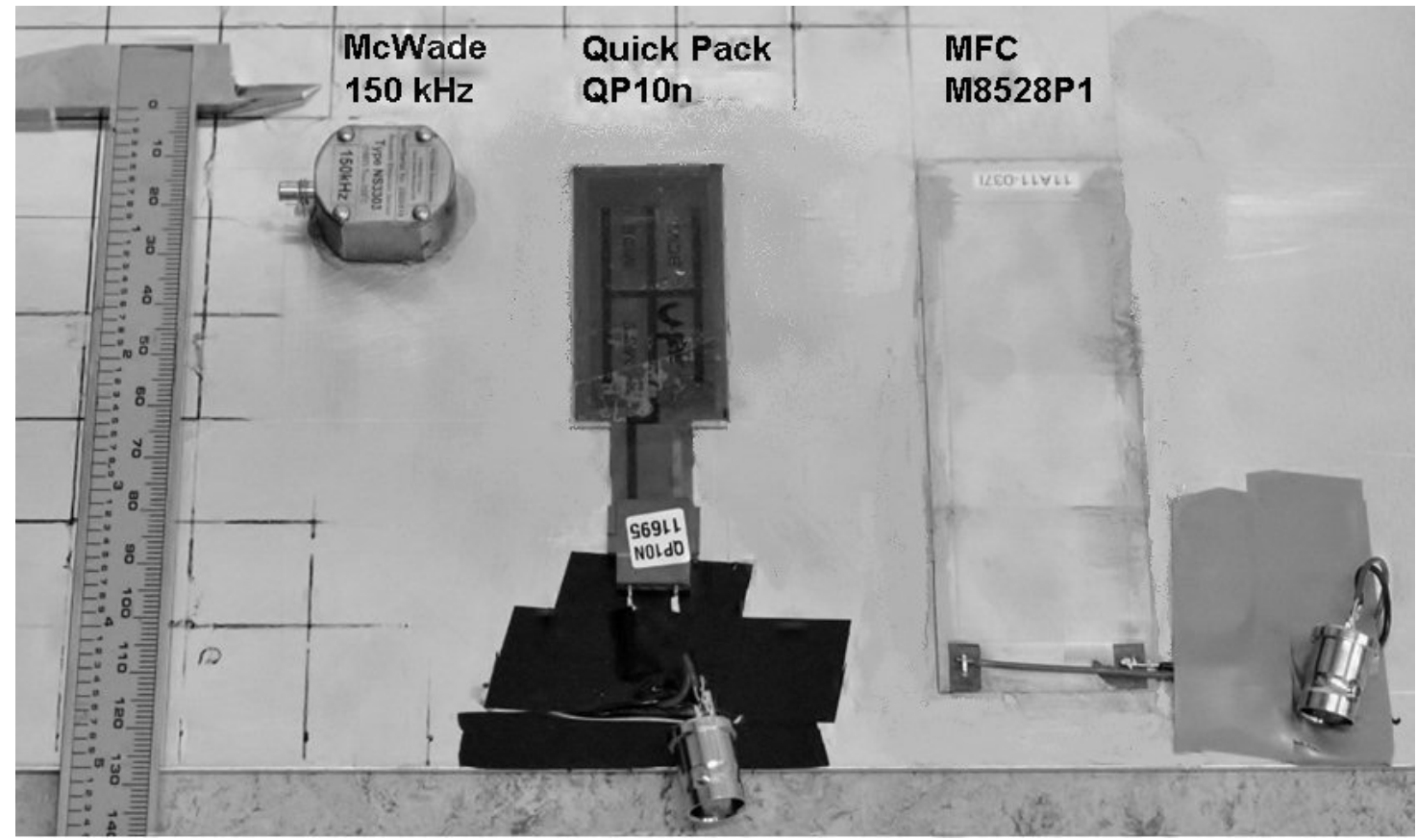

Figure 3. Photograph of the three types of piezoelectric transducers used in the experimental energy transmission system

The remaining two tested transducers were of the patch type. This work is, to the author's knowledge, the first example of the use of such transducers in the ultrasonic energy transmission application. The Mide Quick Pack QP10n transducer is built from a $0.25 \mathrm{~mm}$-thick solid sheet of 
PZT [12]. The dimensions of the sheet are $20 \times 46 \mathrm{~mm}$ (other transducer sizes are available from the manufacturer). The transducer is brittle and cannot be bent without damage. The rigidity of the transducer patch results in the existence of multiple resonances within the investigated frequency range of up to $200 \mathrm{kHz}$. As shown below, this characteristic made the Quick Pack-based energy transmission function efficiently only in narrow frequency ranges.

The Smart Material MFC M8528-P1 is built from multiple parallel PZT fibres in an epoxy resin matrix. The fibres are approximately $180 \mu \mathrm{m}$ tall and $360 \mu \mathrm{m}$ wide and separated from each other by approximately $50 \mu \mathrm{m}$ [13]. Such a composite structure makes the actuator flexible, which is a significant advantage over rigid actuators such as the Quick Pack. The dimensions of the investigated transducer were $85 \times 28 \mathrm{~mm}$ (other sizes are also available). The flexibility of the fibre-based transducer resulted in a lack of resonances, which made the transducer function efficiently across a broad frequency range.

The transmission transducers were driven with an Agilent 33220A signal generator. The drive circuit also comprised an inductor for the compensation of the transducer reactance and a resistor for the current measurement. The signal generator produces voltage waveforms with up to $20 \mathrm{~V}$ peak-to-peak amplitude. Since the maximum drive voltage of the patch transducers is approximately $1000 \mathrm{~V}$ [14], an increase of the drive voltage amplitude is a simple way to increase the system power throughput beyond values reported in this article.

The reception transducers were connected to a circuit comprising a load resistor and a reactance compensation inductor. The voltage drop across the load resistor was used to derive the magnitude of the received electric power. All voltage measurements were made using an Agilent four channel digital oscilloscope.

\section{ENERGY TRANSMISSION MEASUREMENTS}

Energy throughput of three setups, each containing a pair of transducers of one of the previously described types, was measured. The measurement was performed across the frequency range of $20-230 \mathrm{kHz}$. Additional measurements, not reported in this article, showed that the energy throughput maxima of all investigated transducers fell within this range. Furthermore, the $20 \mathrm{kHz}$ lower limit was chosen in order to ensure that the system operated above sound frequencies audible to humans. Recorded power transmission characteristics are shown in Figure 4.
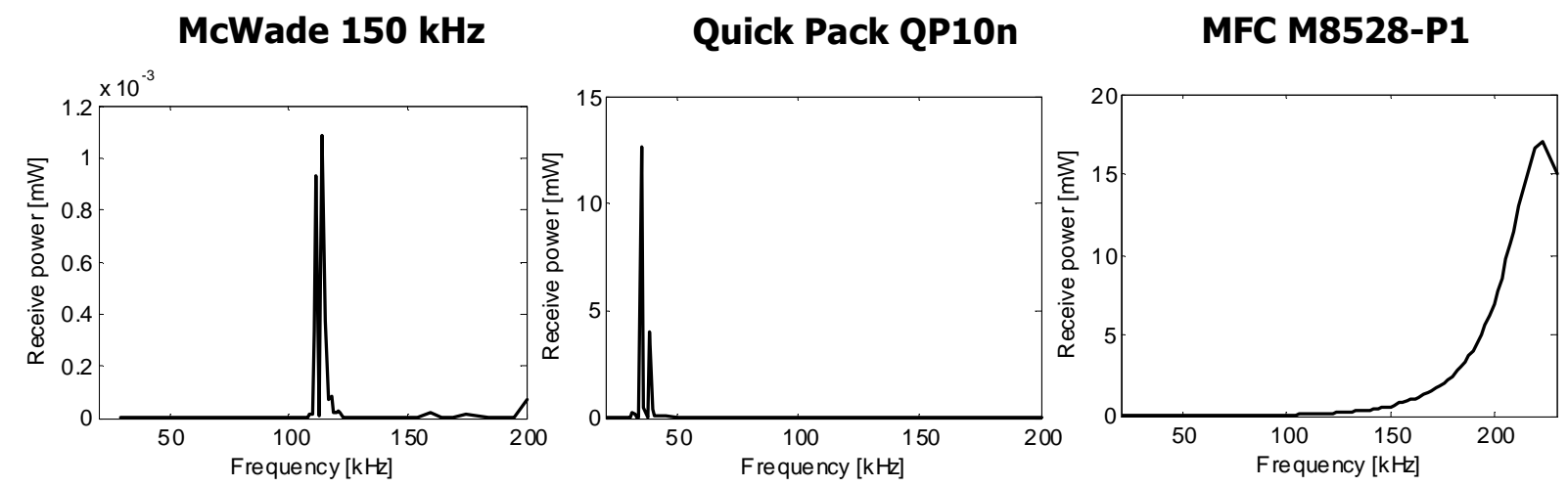

Figure 4. Transmitted power characteristics of investigated setups

The McWade transducers was the least efficient of the investigated types. The setup based on this type transmitted $0.001 \mathrm{~mW}$ of power when driven with the maximum available $20 \mathrm{~V}$ signal. The reported energy throughput occurred only at the $117 \mathrm{kHz}$ resonance frequency and decreased steeply away from it. 
The Quick Pack transducer setup transmitted $13 \mathrm{~mW}$ with $11 \%$ efficiency. The optimum frequency range was also narrow and was centred around $35 \mathrm{kHz}$.

The MFC transducer transmitted $17 \mathrm{~mW}$ with $30 \%$ efficiency. Unlike the previous two transducers, its operating frequency range was wide. The optimum frequency was $224 \mathrm{kHz}$, and the power throughput decreased gradually, reaching $50 \%$ of the maximum at approximately $200 \mathrm{~Hz}$.

The MFC transducer was shown to perform significantly better in the ultrasonic power transmission application than the other two tested transducer types. The power throughput and transmission efficiency achieved with the MFC transducer were the highest of the three tested transducer types. In real-life application, the MFC's wide operating frequency range will make achieving a close-to-optimum operating frequency significantly easier. Also matching transducer examples with slightly differing frequency characteristics will not pose a difficulty. Finally, the adjustment of the operating frequency in order to account for Lamb wave propagation effects (such as the formation of a standing wave, as described in the next section) will be possible to be made without a large decrease in the available maximum power throughput.

\section{LAMB WAVE PROPAGATION MAPPING AND SIMULATION}

The propagation of the Lamb wave in the experimental plate was imaged using a Polytec scanning laser vibrometer. Subsequently, the propagation was modelled using LISA finite difference method software.

Figure 5. shows vibration amplitude maps taken using the vibrometer during the operation of the Quick Pack (left image) and the MFC (right image) transducers. The maximum amplitude of vibration generated by the Quick Pack is $30 \mathrm{~nm}$ and $1.5 \mathrm{~nm}$ when generated by the MFC (the numbers are zero-peak values). Immediately visible are standing wave patterns. These patterns emerge as waves propagating from the transducers and those reflected from the plate edges overlap and interfere. The difference between the patterns generated by the two transducer types correspond to transducer directional characteristics. The Quick Pack-generated pattern is periodic in two perpendicular directions - along and across the transducer length. Directional transmission measurements [15] showed that this transducer generates Lamb waves of similar magnitudes with both its shorter and longer ranges. Waves propagating along two perpendicular axes and their reflections interfere to create the observed pattern.
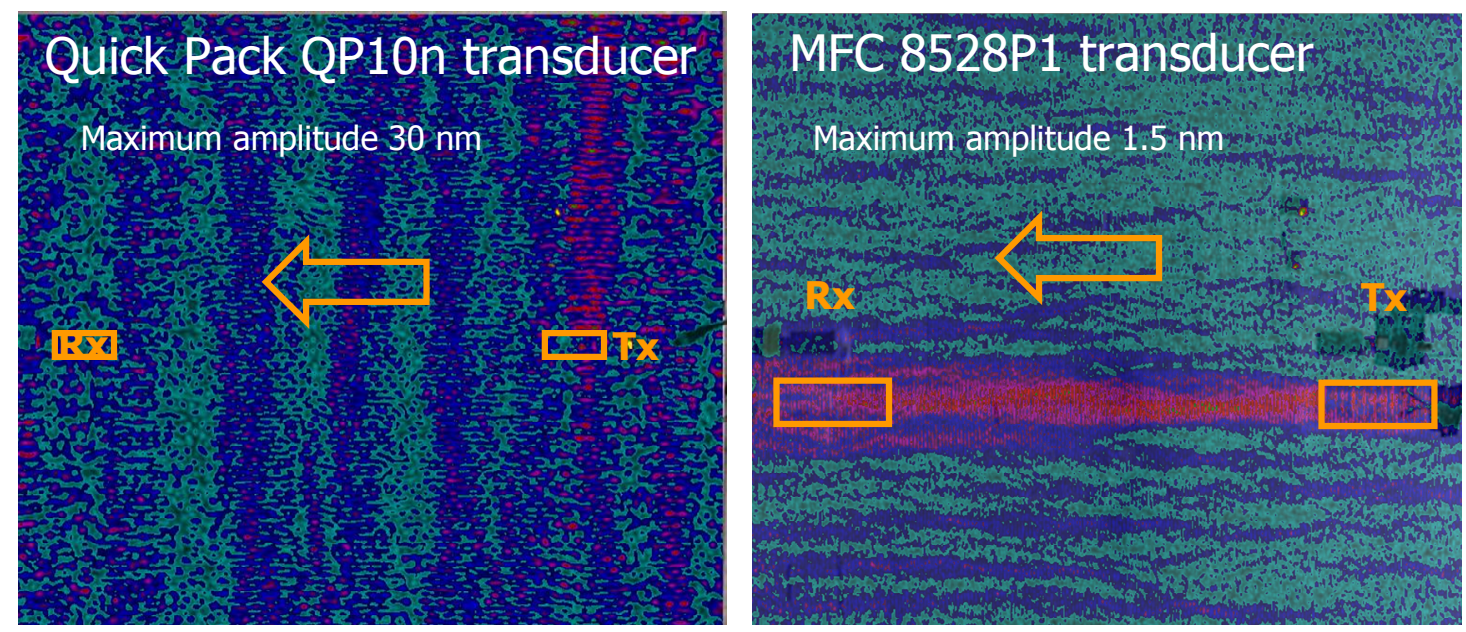

Figure 5. Vibration amplitude cans of the experimental plate during the operation of the Quick Pack and the MFC transducers. Magenta denotes maximum recorded values (30 nm for the Quick Pack and $1.5 \mathrm{~nm}$ for the MFC). Operating frequencies are $65 \mathrm{kHz}$ for the Quick Pack and $181 \mathrm{kHz}$ for the MFC. 
The MFC-generated pattern is periodic in one direction - along the transducer's longer edge. Directional transmission measurements [16] show that this transducer generates Lamb waves only in the direction along its longer edge, which corresponds to the one-directional periodicity of the interference pattern.

The wavelengths of the standing waves are in agreement with the Lamb wave wavelengths expected at the investigated operating frequencies. At $65 \mathrm{kHz}$ (the Quick Pack operating frequency) the theoretically-derived asymmetric $\left(\mathrm{A}_{0}\right)$ Lamb wave mode wavelength in a $1.5 \mathrm{~mm}$ thick aluminium plate is $14.6 \mathrm{~mm}$. The measured standing wave wavelength is $8 \mathrm{~mm}$. This is close to $1 / 2$ of the propagating wave wavelength and is in agreement with the theoretical expectation.

The $\mathrm{A}_{0}$ mode wavelength at $181 \mathrm{kHz}$ (the MFC operating frequency) is $8 \mathrm{~mm}$, and the measured standing wave pattern was $4 \mathrm{~mm}$ - also in agreement with theory.

The symmetric $\left(\mathrm{S}_{0}\right)$ mode amplitudes generated by both transducer types are approximately 10 times smaller than the $\mathrm{A}_{0}$ mode amplitudes [16]. This explains the absence of an $\mathrm{S}_{0}$ mode-related interference patterns in the vibrometer scan.

Figure 6. shows the maps of continuously transmitted Lamb wave propagation in a model of the experimental plate simulated using the LISA software. While the laser vibrometer maps showed amplitudes of vibration of the plate surface (the time and wave phase information was missing), the simulated maps show snapshots of the propagating waves at a particular point in time. The wavelength of the simulated pattern are $17 \mathrm{~mm}$ (for the Quick Pack model at $65 \mathrm{kHz}$ ) and $8 \mathrm{~mm}$ (for the MFC model at $181 \mathrm{kHz}$ ). These values are close to the theoretically-derived $\mathrm{A}_{0}$ mode wavelengths at both frequencies. Overall, the agreement between the measured and simulated Lamb wave propagation patterns is satisfying.
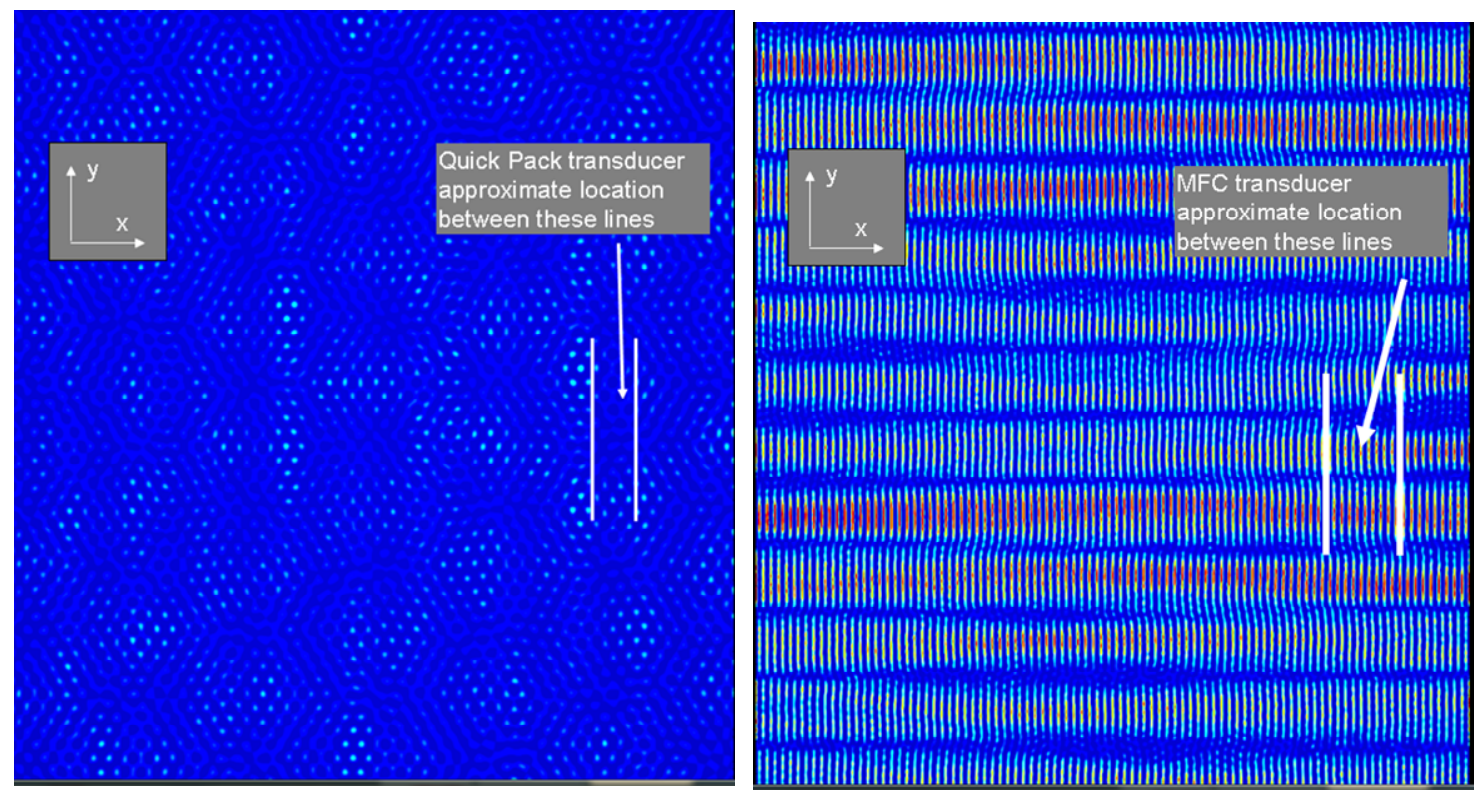

Figure 6. Snapshots of simulated in-plane $x$-axis (horizontal on the illustration) component displacement. Vibration is continuously transmitted by a Quick Pack (left) and MFC M8518-P1 transducer model (right). Snapshots taken 10 ms into simulation

The following information about the design of the system can be drawn from the measured patterns. Firstly, the Lamb wave wavelength is multiple times shorter than the length of the transducer. Such a situation is not favourable, since Lamb waves are generated and received optimally when the length of the transducer is close to $1 / 2$ of the wavelength [17]. This indicated the possibility to optimise the transducer design. Secondly, due to the existence of the standing wave pattern, it is possible that the receiving transducer will be located in an area where the 
vibration amplitude is decreased due to destructive interference. Because the geometry of the standing wave changes with frequency, a frequency tuning system can be envisioned. Such a system could adjust the pattern until the vibration at the receiver location is maximised. The MFC transducer is best suited for the operation with such a system, due to this transducer's wide operating frequency range.

\section{CONCLUSION}

In the reported study the feasibility of the Lamb wave energy transmission method was demonstrated. An experimental setup consisting of a $1.5 \mathrm{~mm}$ thick aluminium plate and pairs of transmitting and receiving transducers was built. The energy transmission distance was $54 \mathrm{~cm}$. Three transducer types were evaluated. The best performing type, the MFC M8528-P1, achieved a power transmission throughput of $17 \mathrm{~mW}$ with $30 \%$ efficiency at $220 \mathrm{kHz}$ frequency while being driven by a $20 \mathrm{~V}$ peak-to-peak voltage. The transmitted power is of an order of magnitude lower than required by the currently developed prototype SHM sensor node. This value, however, can be increased by increasing the drive voltage, which in reported experiments were at $2 \%$ of the transducer maximum.

Imaging of the Lamb wave propagation in the experimental setup plate was performed using a scanning laser vibrometer. The imaged patterns correspond to the theoretical expectation. The patterns were used to derive knowledge about the characteristics of the transmission system and propose improvements to the design. A simulation of the wave propagation was also conducted using the LISA software. The results of the simulation match the experiment.

\section{ACKNOWLEDGEMENTS}

The article presents selected results of research performed as part of the author's $\mathrm{PhD}$ at Cardiff University School of Engineering. The work was supervised by dr Carol Featherston, dr Rhys Pullin, prof. Karen Holford (Cardiff University) and dr. Christophe Paget (Airbus). The project was sponsored jointly by Airbus and Cardiff University.

\section{REFERENCES}

1. Carol A. Featherston; Karen M. Holford; Rhys Pullin; Jonathan Lees; Mark Eaton; Matthew Pearson; An autonomous structural health monitoring solution. Proc. SPIE 8763, Smart Sensors, Actuators, and MEMS VI, 876302 (May 17, 2013); doi:10.1117/12.2018401.

2. D. A. Shoudy, G. J. Saulnier, H. A. Scarton, P. K. Das, S. Roa-Prada, J. D. Ashdown, and A. J. Gavens, "An Ultrasonic Through-Wall Communication System with Power Harvesting," 2007 IEEE Ultrasonics Symposium Proceedings, pp. 1848-1853, Oct. 2007.

3. M. Kluge, T. Becker, J. Schalk, and T. Otterpohl, "Remote acoustic powering and data transmission for sensors inside of conductive envelopes," 2008 IEEE Sensors, Oct. 2008.

4. T. J. Lawry, K. R. Wilt, S. Roa-Prada, J. D. Ashdown, G. J. Saulnier, H. A. Scarton, P. K. Das, and A. J. Gavens, 'A high-temperature acoustic-electric system for power delivery and data communication through thick metallic barriers', in Proceedings of SPIE, vol 8035, p. 80351D-80351D-12. 2011.

5. X. Bao, W. Biederman, S. Sherrit, M. Badescu, Y. Bar-Cohen, C. Jones, J. Aldrich, and Z. Chang, "High-power piezoelectric acoustic-electric power feedthru for metal walls," in Proceedings of SPIE, 2008, vol. 6930, no. 1, p. 69300Z-69300Z-8.

6. S. Moss, J. Skippen, M. Konak, I. Powlesland, and S. Galea, "Detachable acoustic electric feedthrough," in Proceedings of SPIE, 2010, vol. 7647, no. 1, pp. 764745-764745-12. 
7. P. Larson and B. Towe, "Miniature ultrasonically powered wireless nerve cuff stimulator," Neural Engineering (NER), 2011 5th, pp. 265-268, 2011.

8. T. Maleki, N. Cao, S. H. Song, C. Kao, S.-C. A. Ko, and B. Ziaie, "An ultrasonically powered implantable micro-oxygen generator (IMOG).," IEEE transactions on bio-medical engineering, vol. 58, no. 11, pp. 3104-11, Nov. 2011.

9. www.ubeam.com

10. Lamb, H., 1917, "On waves in an elastic plate", in Conference of the Royal Society , London, vol. XCIII, pp. 114-128

11. McWade, Acoustic Emission Solutions, http://www.mcwademonitoring.co.uk/acousticemissions-solutions.php, [retrieved 2013-03-22]

12. Mide, Quick Pack Piezoelectric Transducers Online Catalog, http://www.mide.com/products/qp/qp_catalog.php\#sensor, [retrieved 2013-03-22]

13. Smart Material, n.d., MFC, http://www.smart-material.com/MFC-product-main.html, [retrieved 2013-03-22]

14. Pretorius, J., Hugo, M. \& Spangler, R., 2004. A Comparison of Packaged Piezoactuators for Industrial Applications substrate piezo substrate. Mide Technology Corporation Publication, pp.1-12.

15. Kural, A., Pullin, R., Holford, K., Lees, J., Naylon, J., Paget, C. and Featherston, C. 2013. Design and characterization of an ultrasonic lamb-wave power delivery system. Ultrasonics, Ferroelectrics and Frequency Control, IEEE Transactions on 60(6)

16. Kural, A., 2013, Ultrasonic Lamb Wave Energy Transmission System for Aircraft Structural Health Monitoring Applications. PhD thesis at Cardiff University.

17. Haig, A. 2010. MFC Presentation (SHeMS Meeting Jul 2010). 